\title{
Optimal generator rescheduling with distributed slack bus model for congestion management using improved teaching learning based optimization algorithm
}

\author{
S T SUGANTHI ${ }^{1, *_{0}}$, D DEVARAJ ${ }^{2}$, S HOSIMIN THILAGAR $^{3}$ and K RAMAR ${ }^{4}$ \\ ${ }^{1}$ Department of EEE, Sri Shakthi Institute of Engineering and Technology, Coimbatore, India \\ ${ }^{2}$ Department of EEE, Kalasalingam University, Krishnan koil, India \\ ${ }^{3}$ Department of EEE, Anna University, Chennai, India \\ ${ }^{4}$ Faculty of Engineering, Multimedia University, Cyberjaya, Malaysia \\ e-mail: suganthi.sb@gmail.com
}

MS received 13 October 2017; revised 17 March 2018; accepted 1 May 2018; published online 1 October 2018

\begin{abstract}
This article proposes a new strategy for Congestion Management (CM) through Generation Rescheduling (GR) with Distributed Slack Bus (DSB) model using Improved Teaching Learning Based Optimization (ITLBO) algorithm. Most of the previous research mainly focuses on rescheduling the existing generators for $\mathrm{CM}$ and least concerned about the consequence of this rescheduling on the slack bus. As a result, the slack bus is supposed to carry the entire residual effect of all other generators which leads to increased Congestion Management Cost (CMC). This work proposes a distributed slack bus model for reducing the excess burden on the slack bus and a two-fold contribution is made to develop the proposed technique. First, is the selection of the participating generator for CM by means of Incremental Generator Sensitivity Factor (IGSF), which adopts the generator sensitivity as well as the bidding cost. Next, CM is formulated as an optimization problem with the objective function involving congestion management cost and solved using ITLBO algorithm, which incorporates the self-motivated learning concept with the basic TLBO operators. For evaluation, the standard IEEE 30-bus and IEEE 118-bus systems are used and the obtained results are compared with the other CM systems reported through literature.
\end{abstract}

Keywords. Congestion management; generation rescheduling; generator sensitivity factor; teaching learning based optimization; restructured power systems.

\section{Introduction}

Due to transmission open access in the deregulated electricity market, the power system often operates very close to or even beyond their security limits because of increased, unexpected power exchanges as each market operator always tries to get maximum profit. If these unexpected power exchanges are not restricted by the appropriate way, overloading in one or more transmission lines may occur leading to a condition called congestion. Hence, CM is one of the key functions of any system operator ( $\mathrm{SO}$ ) in the deregulated electricity market. Based on the bids submitted by the generators and loads, the SO clears the market with suitable market clearing mechanism [1,2], which results in the price to be charged by the generator and paid by the loads for the next 24 hours. Also, the SO has the sole responsibility to ensure a secure system operation and resorts to minimal system changes if congestion occurs.

*For correspondence
In such cases, both the generators and loads may have to alter their schedules from the market clearing values to contribute to congestion management.

Rescheduling of generators [3, 4], load curtailment [5-7], usage of regulating transformers/tap setting transformers [8], FACTS devices, etc., [9, 10] are the proposed techniques to relieve congestion. SO generally prefers to use the rescheduling of generators instead of any other techniques so that the system topology remains unaltered. In all these literature, it is assumed that the changes in generations and loads employed for CM are exactly compensated by corresponding changes in a generation at the slack bus. This may introduce large changes in the slack bus power generation which lead to increased congestion management cost (CMC).

This paper proposes a technique to reduce the excess burden on the slack bus using Power Distribution Factor (PDF) $[11,12]$, through which the real power adjustment in any one of the generator buses can be distributed to all other generator buses while alleviating the congestion. The 
proposed Generator Sensitivity Factor (GSF) which is derived with the help of power distribution factor helps in identifying the most sensitive generators for the rescheduling process. The real power adjustment in the identified buses does not reflect on the slack bus which enhances a further reduction in congestion management cost. Also, a novel strategy is adopted which combines the sensitivity and bidding cost, for selection of participating nodes.

Recently, the advancements in computation technology like parallel computation have stimulated many recent researchers to focus on the application of artificial intelligent (AI) techniques for CM problems in restructured power systems. Meta-heuristics algorithms like genetic algorithms (GA) [13, 14], Particle Swarm Optimization (PSO) $[15,16]$, etc., are applied to find the optimal settings of real power values which result in minimum congestion cost. The application of Differential Evolution (DE) for CM is discussed in [17, 18]. An attempt is made in [4], to improve the convergence of DE with DBMO (Double Best Mutation Operator) for CM.

Rao et al proposed a new optimization technique which is called as Teaching-Learning Based Optimization (TLBO) algorithm, found to be more efficient in solving constrained and large scale optimization problems [19, 20]. This article explores the ability of TLBO algorithm for CM problems. TLBO is motivated by teaching learning process of a class room. In this work, a concept called self-motivated learning is combined with the existing operators to improve the performance of TLBO. The major contributions of this article are:

(i) Development of a novel Generator Sensitivity Factor (GSF) using network participation factor to effectively identify the participating generators, which helps to reduce the burden on the slack bus during the rescheduling process of congestion management.

(ii) Establishment of a novel strategy which combines the generator sensitivity and bidding cost to ensure the minimum congestion cost.

(iii) Development of ITLBO based optimization algorithm for obtaining minimum congestion management cost.

(iv) Exhibit the superiority of the proposed ITLBO algorithm with the other algorithms for CM problem.

This work illustrates the feasibility of the proposed approach by testing standard IEEE 30-bus system and IEEE 118-bus systems. The article is organized as follows. Section 2 discusses in detail the CM problem and the derivation of sensitivity factors for identification of participating generators. Section 3 describes the overview of TLBO and section 4 describes the proposed ITLBO algorithms. Section 5 elaborates the development of the proposed approach using ITLBO algorithm. The results showing the feasibility of the proposed method are discussed in section 6. The major contributions and conclusions are presented in section 7 .

\section{Congestion management - problem formulation}

In day-ahead electricity markets, the SO clears the market using suitable market clearing mechanism [1, 2] based on the bids submitted by generators and loads for 24 hours. This results in hourly prices to be paid by the loads and to be charged by the generators. In case of any transmission line congestion, the SO does the CM hour by hour. Here CM problem is formulated as a nonlinear optimization problem with the objective of minimizing the congestion management cost using Generator Rescheduling with the time frame of one hour. The first step in the CM is the selection of the participating generators for rescheduling process and the next step is formulation and solving the optimization problem. The method for selecting the participation generator has been explained in the first part of this section and the formulation of the optimization problem is done in the second part.

\subsection{Sensitivity factor for identification of participating generator}

The sensitivity factor introduced here is to effectively identify the participating generators for rescheduling to alleviate the congestion as all the generators in the system may not have the same sensitivity to the power flow in congested lines. The Power Distribution Factor $(\gamma)$ introduced here is used to distribute the concentrated real power on the slack bus due to the rescheduling process among all other generator buses. The incremental sensitivity factor proposed here is used to achieve congestion alleviation with minimum cost. The proposed Generator Sensitivity Factor (GSF) and Incremental Generator Sensitivity Factor (IGSF) are derived as follows.

2.1a Generator sensitivity factors: Assume there are $\mathrm{n}_{\mathrm{g}}$ a number of generator buses with bus 1 as slack bus. A change in real power flow through line $k$ connected between buses $i$ and $j$ due to a change in real power injection by a generator connected to bus $g$ is called the Generator Sensitivity Factor. It is expressed as follows,

$$
\mathrm{GSF}=\frac{\Delta \mathrm{P}_{\mathrm{ij}}}{\Delta \mathrm{P}_{\mathrm{Gg}}}
$$

Expanding Eq. (1) after neglecting P-V coupling,

$$
G S F=\frac{\partial P_{i j}}{\partial \delta_{i}} \cdot \frac{\partial \delta_{i}}{\partial P_{G g}}+\frac{\partial P_{i j}}{\partial \delta_{j}} \cdot \frac{\partial \delta_{j}}{\partial P_{G g}}
$$


The active power flow through the transmission line can be written as,

$$
P_{i j}=-V_{i}^{2} G_{i j}+V_{i} V_{j} G_{i j} \cos \left(\delta_{i}-\delta_{j}\right)+V_{i} V_{j} B_{i j} \sin \left(\delta_{i}-\delta_{j}\right)
$$

Differentiating Eq. (3) with respect to $\delta_{i}$ and $\delta_{j}$, we obtain,

$$
\begin{gathered}
\frac{\partial P_{i j}}{\partial \delta_{i}}=-V_{i} V_{j} G_{i j} \sin \left(\delta_{i}-\delta_{j}\right)+V_{i} V_{j} B_{i j} \cos \left(\delta_{i}-\delta_{j}\right) \\
\frac{\partial P_{i j}}{\partial \delta_{j}}=+V_{i} V_{j} G_{i j} \sin \left(\delta_{i}-\delta_{j}\right)-V_{i} V_{j} B_{i j} \cos \left(\delta_{i}-\delta_{j}\right) \\
=-\frac{\partial P_{i j}}{\partial \delta_{i}}
\end{gathered}
$$

The values of $\frac{\partial P_{i j}}{\partial \delta_{i}}$ and $\frac{\partial P_{i j}}{\partial \delta_{i}}$ can are obtained from Eqs. (4) and (5). Here a change in $\mathrm{g}^{\text {th }}$ generator is compensated by a negative change in all other buses proportional to their maximum MW rating except slack bus. The proportional real power changes at any generator bus for a change in $\Delta P_{G g}$ is called Power Distribution Factor $(\gamma)$ given by,

$$
\gamma_{m}=\frac{P_{m}^{\max }}{\sum_{\substack{k=1 \\ k \neq j}}^{n_{g}} P_{k}^{\max }} \quad k=1,2, . . n_{g}
$$

Now the active power at any generator bus except the slack bus due to a change in real power $\Delta P_{G g}$ at $\mathrm{g}^{\text {th }}$ generator can be,

$$
\Delta P_{G m}=-\left(\gamma_{m} \times \Delta P_{G g}\right) \quad \text { where } m=1,2,3, . . n_{g} \& m \neq g
$$

To implement this power distribution factor a modified Jacobian matrix is considered which includes the slack bus elements in its matrix. This can be done as follows. Hence the new matrix looks like,

$$
\left[\begin{array}{r}
\Delta P_{1} \\
\Delta P_{2} \\
\cdot \\
\cdot \\
\cdot \\
\Delta P_{n g}
\end{array}\right]=\left[\begin{array}{lll}
\frac{\partial P_{1}}{\partial \delta_{1}} \frac{\partial P_{1}}{\partial \delta_{2}} \cdots \frac{\partial P_{1}}{\partial \delta_{n g}} & -\gamma_{1} \\
\frac{\partial P_{g}}{\partial \delta_{1}} \frac{\partial P_{g}}{\partial \delta_{2}} \cdots \frac{\partial P_{g}}{\partial \delta_{n g}} & -\gamma_{2} \\
& \cdot \\
\frac{\partial P_{n g}}{\partial \delta_{1}} \frac{\partial P_{n g}}{\partial \delta_{2}} & \cdots \frac{\partial P_{n g}}{\partial \delta_{n g}} & -\gamma_{n g}
\end{array}\right]\left[\begin{array}{r}
\Delta \delta_{1} \\
\Delta \delta_{2} \\
\cdot \\
\cdot \\
\cdot \\
\Delta \delta_{n g} \\
\Delta P_{G g}
\end{array}\right]
$$

The extra column added in the Jacobian is due to change in active power due to power distribution factor. Now at any generator bus, the power flow equation becomes,

$$
P_{i}=\sum_{j=1}^{n_{g}} V_{i} V_{j} Y_{i j} \cos \left(\theta_{i j}-\delta_{i}+\delta_{j}\right)+\left(-\gamma_{i} \Delta P_{G_{g}}\right)
$$

Hence from Eq. (9), the values $\frac{\partial \delta_{i}}{\partial P_{G_{g}}}$ and $\frac{\partial \delta_{j}}{\partial P_{G_{g}}}$ can be obtained.

2.1b Incremental generator sensitivity sactors: As the objective is to alleviate the congestion with minimum congestion management cost, generators with low bidding cost and high sensitivity are selected. For this purpose, a factor called Incremental Generator Sensitivity Factor $(I G S F)$ is proposed here and defined as follows:

$$
I G S F=\left\{\begin{array}{lll}
\frac{R_{g}^{d}}{G S F} & \text { if } & G S F>0 \\
\frac{R_{g}^{u}}{G S F} & \text { if } & G S F<0
\end{array}\right.
$$

The generator having low values of $I G S F$ will meet the both requirements stated earlier. Hence it is decided to select the generators having low values of IGSF for the rescheduling process. The participating generator can either increase or decrease its real power generation for congestion alleviation depends on the sign of $I G S F$ value.

\subsection{Problem formulation}

In $\mathrm{CM}$ the $\mathrm{SO}$ has the responsibility for the amount of active power that should be rescheduled for the congestion alleviation. While doing so, the bids submitted by generating units must be considered in order to maximize the social benefit. Hence the main objective of congestion is to minimize the rescheduling cost based on the bids submitted by each Generating Unit. The objective function of the optimization problem is to minimize congestion management cost,

$$
C M C=\sum_{g=1}^{n_{g}}\left(R_{g}^{u} \Delta P_{G g}^{u}+R_{g}^{d} \Delta P_{G g}^{d}\right)
$$

Subject to,

2.2a Constraints on decision / independent variables:

$$
\begin{gathered}
\Delta P_{G g}^{u}, \Delta P_{G g}^{d} \geq 0 \\
P_{G g}=P_{G g}^{0}+\Delta P_{G g}^{u} \text { or } P_{G g}=P_{G g}^{0}-\Delta P_{G g}^{d} \\
P_{G g}^{\min } \leq P_{G g} \leq P_{G g}^{\max } \\
\sum_{g=1}^{n_{g}} \Delta P_{G g}=0 \quad \text { where } g=1,2, \ldots n_{g}
\end{gathered}
$$

Here $P_{G g}^{0}$ and $F_{k}^{0}$ are the output of market clearing procedure.

2.2b Constraints on dependent variables: Load bus Voltage Constraint: 


$$
V_{L l}^{\min } \leq V_{L l} \leq V_{L l}^{\max }
$$

2.2c Constraint on generation reactive power:

$$
Q_{G g}^{\min } \leq Q_{G g} \leq Q_{G g}^{\max }
$$

2.2d Transmission line apparent power flow limit:

$$
F_{k} \leq F_{k}^{\max }
$$

The main objective here is to obtain the minimum $\mathrm{CMC}$, at the same time satisfying constraints on all the independent and dependent variables. Involving all these constraints the original objective function as can be written as

$$
O F=C M C+\sum_{j=1}^{n l} V P_{j}+\sum_{j=1}^{n g} Q P_{j}+\sum_{j=1}^{n k} S F_{j}
$$

Here $V P_{j}, Q P_{j}$ and $S F_{j}$ are the penalty terms for load bus voltage limit violation, reactive power generation limit violations and line apparent power flow limit violations, respectively. They can be defined as,

$$
\begin{gathered}
V P_{j}=\left\{\begin{array}{cl}
K_{v}\left(V_{j}-V_{j}^{\max }\right)^{2} & \text { if } V_{j}>V_{j}^{\max } \\
K_{v}\left(V_{j}-V_{j}^{\min }\right)^{2} & \text { if } V_{j}<V_{j}^{\min } \\
0 & \text { otherwise }
\end{array}\right. \\
Q P_{j}=\left\{\begin{array}{cl}
K_{q}\left(Q_{j}-Q_{j}^{\max }\right)^{2} & \text { if } Q_{j}>Q_{j}^{\max } \\
K_{q}\left(Q_{j}-Q_{j}^{\min }\right)^{2} & \text { if } Q_{j}<Q_{j}^{\min } \\
0 & \text { otherwise }
\end{array}\right. \\
S F_{j}=\left\{\begin{array}{cl}
K_{f}\left(F_{j}-F_{j}^{\max }\right)^{2} & \text { if } F_{j}>F_{j}^{\max } \\
0 & \text { otherwise }
\end{array}\right.
\end{gathered}
$$

Proper selection of the penalty parameters $K_{v}, K_{q}$ and $K_{f}$ plays a key role in obtaining the optimal solution. The above said CM problem is solved using ITLBO algorithm in this work.

\section{Overview of Teaching Learning based Optimization algorithm}

TLBO algorithm is a recently evolved population based optimization algorithm, suggested by Rao et al [19]. The TLBO algorithm is motivated by teaching-learning process of a classroom. Each learner in the class represents a candidate solution and the different courses offered represents the decision variables in TLBO. The algorithm considers two different modes of learning; (i) learning from the teacher (Teacher phase), (ii) learning by interaction among the learners (Learning phase). The grades/marks obtained by the learners are treated as the output of the TLBO algorithm. The results obtained by the learners are comparable to the fitness of optimization problem.

\subsection{Teacher phase}

In this phase, the mean learning value of the class is increased by imparting knowledge to the learners by the teacher using effective teaching methodologies. For that purpose, the teacher should analyze the results of the learners how far it is deviating from the targeted result, then tries to improve the results by appropriate teaching method. This analysis is done by,

$$
\operatorname{Diff} \_M e a n_{i}=\operatorname{rand}_{i}\left(M_{\text {new }}-T_{F} M_{i}\right)
$$

where $M_{i}$ is the mean result of the learners at any instant i, and $M_{n e w}$ is the targeted new mean of the learners, $T_{F}$ is the teaching factor which decides the value of mean either be 1 or 0 and rand $_{i}$ is random number in the range varying from 0 to $1 . T_{F}$ is defined by,

$$
T_{F}=\operatorname{round}[1+\operatorname{rand}(0,1) \times(2-1)]
$$

Then the learners can be updated by,

$$
X_{\text {new }, i}=X_{\text {old }, i}+\text { Diff_Mean }
$$

\subsection{Learner phase}

The interaction among the learners always enhances the knowledge level of learners. This is the main phenomenon behind the phase. The mathematical expression of this phase is,

$$
X_{n e w, i}=\left\{\begin{array}{lll}
X_{\text {old }, i}+\operatorname{rand}_{i}\left(X_{i}-X_{j}\right) & \text { if } & f\left(X_{j}\right)>f\left(X_{i}\right) \\
X_{\text {old }, i}+\operatorname{rand}_{i}\left(X_{j}-X_{i}\right) & \text { if } & f\left(X_{i}\right)>f\left(X_{j}\right)
\end{array}\right.
$$

The learner $X_{i}$ gets new information if the learner $X_{j}$ has more knowledge than $X_{i}$ according to the above equation. If the new solution $X_{\text {new }}$ is better, it is selected for next iteration.

\section{Improved teaching learning based optimization algorithm}

In the basic TLBO algorithm, the result of any learner is assessed based on the knowledge imparted by the teacher and the knowledge gained from the fellow learners. But every learner must possess self-learning ability, based on their own capacity to obtain good results. Hence, the selflearning mechanism of learners is considered here for improved performance. For that purpose, the learning phase in the basic TBLO algorithm is divided into two parts namely (i) Learning from fellow mates and (ii) Selflearning mechanism as follows. 


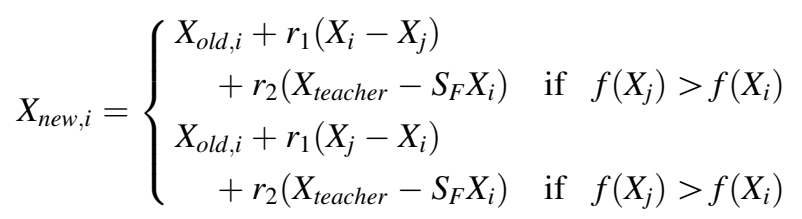

Here $r_{1}$ and $r_{2}$ are the random number in the range $[0,1]$. And $S_{F}$ is known as self-learning factor and defined by,

$$
S_{F}=\operatorname{round}[1+\text { rand }]
$$

With this modification, the performance of TLBO might improve.

\section{Implementation of the proposed method for $\mathrm{CM}$ problem}

The implementation of ITLBO based algorithm for CM based on generation rescheduling is given below. Also the flow chart is illustrated in figure 1 .

Step 1: Perform AC contingency power flow to identify the congested lines.

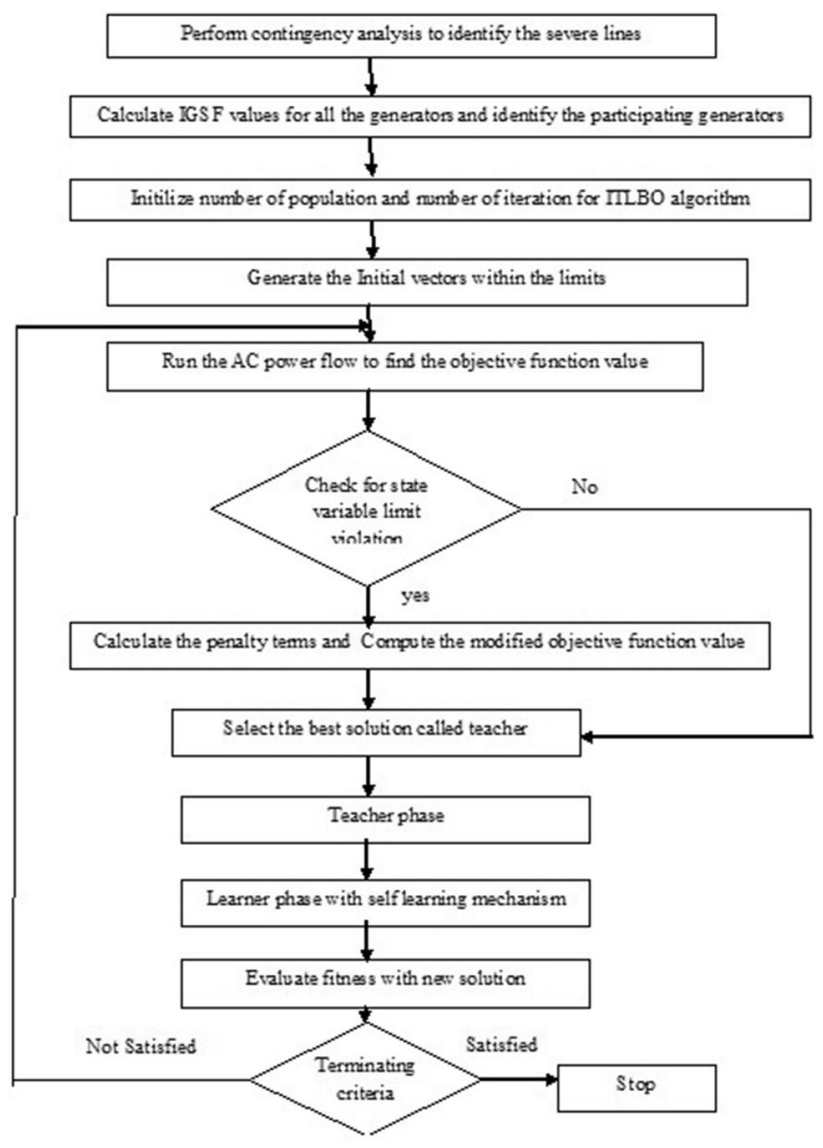

Figure 1. Implementation of ITLBO algorithm.
Step 2: Find the generator sensitivity factors GSF and incremental generator sensitivity factors IGSF for all the congested lines.

Step 3: Based on the computed IGSF values identify the participating generators in which, the real power of generators has to be changed $\left(\Delta P_{G g}\right)$ for alleviating the congestion.

Step 4: Apply ITLBO algorithm. Generate the initial vector $\left(X_{i}^{0}\right)$ by defining a suitable population size, for all the decision variables. Each individual in the initial vector will be represented by,

$$
X_{i}^{0}=\left[\Delta P_{G 1}, \Delta P_{G 2} \ldots \Delta P_{G s g}\right]
$$

The subscripts 'Gsg' refers to the number of generators selected for the real power adjustments, respectively. The initial vector is generated randomly within the permissible limits of the decision variables.

Step 5: Run the AC power flow with the vector $\left(X_{i}^{0}\right)$ for obtaining the reactive powers of generators, voltage magnitudes of all load buses, and line flows.

Step 6: Calculate the penalty terms using Eqs. (21) (23). (The penalty terms will become zero if there is no violation in the corresponding variables.)

Step 7: Compute the modified objective function value of each individual as stated in Eq. (20).

Step 8: Select the best solution among all individuals called Teacher

Step 9: Calculate the mean value of each individual and update the individuals based on Eq. (26) in Teacher Phase.

Step 10: Randomly select any two solutions from the population, and adapt them by evaluating its fitness value with each other (Learner Phase). And generate new population vector considering the self-learning mechanism using Eq. (28).

Step 11: Evaluate the new population and compare with the existing population. If it is better to accept the new population otherwise keep the existing population.

Step 12: Repeat the process from step 5 until the predefined maximum iteration or the convergence criterion gets satisfied.

\section{Numerical example}

In order to evaluate the proposed CM method, simulation tests are carried out on the standard IEEE 30-bus system and IEEE 118-bus system. The proposed ITLBO based approach is implemented in MATLAB using MATPOWER toolbox to obtain minimum congestion management cost.

\subsection{IEEE 30-bus test system}

The details of the IEEE 30-bus test system are given in [4]. The bids submitted by generators and the loads to alter their schedule are given in table 1 . The power flow through the 
congested line for the simulated contingency is given in table 2 .

The obtained generator sensitivity factors and incremental generator sensitivity factors for the congested line are listed in table 3 . Normally, positive values of the generator sensitivity factor indicate that decrease in a generation will decrease the power flow in the congested lines whereas the negative values of the generator sensitivity factor indicate that, increase in generation will decrease the power flow in the congested lines. It can also be noted that the sensitivities corresponding to different generators are very close to each other as the system is very small and connected very tightly together. Hence, it is decided to reschedule all the generators for alleviating the congestion irrespective of IGSF values.

6.1a Calculation of congestion management cost and rescheduling power: After selecting the participating generators the rescheduling process has been done by ISO from the market clearing procedure. The proposed ITLBO based approach is implemented to find the congestion cost and active power rescheduling of IEEE 30-bus test system. In order to find the optimal parameters of the ITLBO algorithm, the number of learners has been varied from 20

Table 1. Generator bidding co-efficients for IEEE 30 bus test system.

\begin{tabular}{lcc}
\hline & \multicolumn{2}{c}{ Bids submitted by generators in $\$$ /MWhr } \\
\cline { 2 - 3 } Generator no. & $R_{g}^{u}$ & $R_{g}^{d}$ \\
\hline 1 & 15 & 11 \\
2 & 17 & 8 \\
5 & 19 & 8 \\
8 & 20 & 12 \\
11 & 15 & 10 \\
13 & 10 & 5 \\
\hline
\end{tabular}

Table 2. Line flow on the congested line for IEEE-30 bus test system.

\begin{tabular}{lccc}
\hline $\begin{array}{l}\text { Congested } \\
\text { line }\end{array}$ & $\begin{array}{c}\text { Line flow } \\
\text { (MVA) }\end{array}$ & $\begin{array}{c}\text { Maximum limit } \\
\text { (MVA) }\end{array}$ & $\begin{array}{c}\text { Violation } \\
\text { (MVA) }\end{array}$ \\
\hline $1-2$ & 175.05 & 130 & 45.05 \\
\hline
\end{tabular}

Table 3. GSF and IGSF values on the congested line (1-2) in IEEE-30 bus test system.

\begin{tabular}{lcrrrr}
\hline $\begin{array}{l}\text { Generator } \\
\text { bus Number }\end{array}$ & 2 & \multicolumn{1}{c}{5} & \multicolumn{1}{c}{8} & \multicolumn{1}{c}{11} & \multicolumn{1}{c}{13} \\
\hline$G S F$ & -0.78 & 0.78 & 0.70 & -0.73 & 0.75 \\
$I G S F$ & -21.5 & -24.15 & -28.32 & -20.36 & -13.23 \\
\hline
\end{tabular}

to 100 in steps of 20 and the number of iteration has been varied from 10 to 100 in steps of 10 . Figure 2 represents the obtained objective function values with different TLBO parameters. From the obtained objective function values, the preferred TLBO parameters for this optimization problem are; (i) Population Size: 20 and (ii) Maximum Iterations: 50.

The optimal results obtained using ITLBO algorithm are listed in table 4. With the Distributed Slack Bus (DSB) model the congestion cost significantly got reduced to $1170.71 \$ / \mathrm{h}$ and also it is very clear that the enduring effect on the slack bus due to the rescheduling process is nil. This cost is nearly $20 \%$ less than the cost incurred by the regular GR process. Further, the slack bus is adjusted by only 2.23 MW whereas in the original case, it needs to be adjusted up to $54 \mathrm{MW}$. The convergence characteristic of the proposed ITLBO method is shown in figure 3, from which one could see the effect of DSB model on the CMC from the first iteration onwards. The amount of rescheduling power on every participating generator is depicted in figure 4 . The real power losses of the system also got reduced with the DSB model.

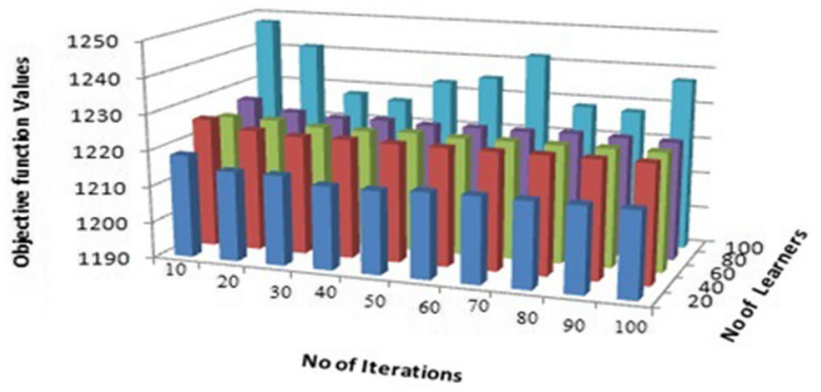

Figure 2. Objective function values for different parameter settings of ITLBO for IEEE 30-bus test system.

Table 4. Optimal settings of decision variables and congestion cost obtained by ITLBO algorithm for IEEE-30 bus test system

\begin{tabular}{|c|c|c|}
\hline Control variables & $\begin{array}{l}\text { GR without } \\
\text { distributed slack bus }\end{array}$ & $\begin{array}{c}\text { GR with } \\
\text { distributed slack } \\
\text { bus }\end{array}$ \\
\hline$\Delta \mathrm{P}_{2}(\mathrm{MW})$ & +20.2 & +25.61 \\
\hline$\Delta \mathrm{P}_{5}(\mathrm{MW})$ & +16.2 & -27.65 \\
\hline$\Delta \mathrm{P}_{8}(\mathrm{MW})$ & +3.3 & -16.68 \\
\hline$\Delta \mathrm{P}_{11}(\mathrm{MW})$ & +8.4 & +15.99 \\
\hline$\Delta \mathrm{P}_{13}(\mathrm{MW})$ & +4.9 & +4.96 \\
\hline $\begin{array}{l}\text { (Slack bus Power) } \\
\Delta \mathrm{P}_{1}(\mathrm{MW})\end{array}$ & -54 & -2.23 \\
\hline $\begin{array}{l}\text { MVA flow on the } \\
\text { congested line }\end{array}$ & 127.66 & 129.71 \\
\hline $\begin{array}{l}\text { Congestion } \\
\text { Management Cost } \\
(\$ / \mathrm{h})\end{array}$ & 1486.2 & 1170.71 \\
\hline $\begin{array}{l}\text { Real power loss } \\
\text { (MW) }\end{array}$ & 11.512 & 11.45 \\
\hline
\end{tabular}




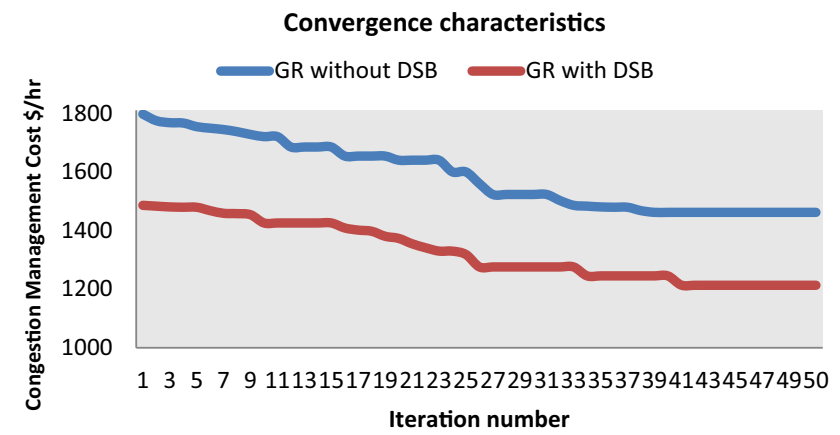

Figure 3. Convergence characteristics of ITLBO with and without DSB model for IEEE 30-bus system.

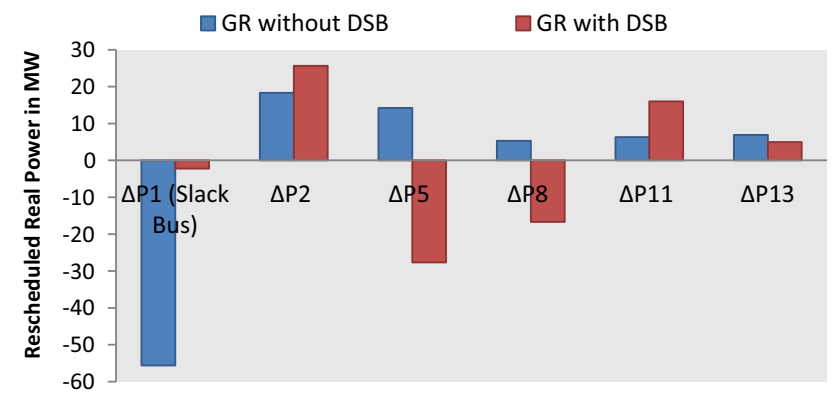

Figure 4. Rescheduled real power with and without DSB model for IEEE 30-bus test system.

6.1b Comparison with other heuristic algorithms: In order to demonstrate the superiority of the proposed ITLBO algorithm in solving CM problem, it is compared with other heuristic algorithms like Genetic Algorithm (GA) [9], Particle Swarm Optimization (PSO) [11], Differential Evolution (DE) [12] algorithm, Improved Differential Evolution (IDE) [4], and conventional TLBO Algorithm. All the algorithms were implemented on the MATLAB platform. The specific parameters of other optimization algorithms used for the simulation are listed in table 5 .

In tables 6 and 7, the calculation of rescheduling power and congestion cost obtained by ITLBO with and without incorporation of DSB has been compared with other algorithms. Both the decision variables and congestion cost have been reported. It is observed that the rescheduling cost obtained by ITLBO is minimum in both scenarios when compared to other algorithms. The congestion cost obtained by the proposed ITLBO is $\mathbf{1 4 8 6 . 2} \$ / \mathbf{h}$ and 1170.71 $\$ / \mathbf{h}$ for without and with the incorporation of DSB, respectively. This is approximately $10 \%$ less when compared to the cost obtained with GA. Also, the convergence characteristics of the ITLBO demonstrates that the objective function value gradually decreases with the number of iterations and reached the best minimum value within 40 iterations. Hence, the proposed ITLBO algorithm is successful in solving CM problem and capable of producing the best results than the other algorithms.
Table 5. Parameters of Implemented heuristics algorithms.

\begin{tabular}{|c|c|c|c|}
\hline Algorithm & Parameters used & Algorithm & Parameters used \\
\hline \multirow[t]{9}{*}{ GA } & Population size : & \multirow[t]{9}{*}{ PSO } & Population size :50 \\
\hline & 30 & & Max inertia weight \\
\hline & Crossover & & $: 0.9$ \\
\hline & Probability : 0.8 & & Min inertia weight \\
\hline & Mutation & & $: 0.4$ \\
\hline & Probability : & & Acceleration \\
\hline & 0.01 & & Constants \\
\hline & Maximum Number & & $\left(\mathrm{C}_{1}, \mathrm{C}_{2}\right): 2$ \\
\hline & of Iterations : 50 & & $\begin{array}{l}\text { Maximum Number } \\
\text { of Iterations : } 50\end{array}$ \\
\hline \multirow[t]{9}{*}{$\mathrm{DE}$} & Population size : & \multirow[t]{9}{*}{ IDE } & Population size :50 \\
\hline & 50 & & Scaling Factor $\left(\mathrm{C}_{1}\right)$ \\
\hline & Scaling Factor $(\mathrm{F})$ : & & $: 2$ \\
\hline & 0.8 & & Scaling Factor $\left(\mathrm{C}_{2}\right)$ \\
\hline & Crossover & & $: 2$ \\
\hline & Constant $\left(\mathrm{C}_{\mathrm{R}}\right)$ : & & Crossover \\
\hline & 0.8 & & Constant $\left(\mathrm{C}_{\mathrm{R}}\right)$ \\
\hline & Maximum Number & & :0.8 \\
\hline & of Iterations : 50 & & $\begin{array}{l}\text { Maximum Number } \\
\text { of Iterations : } 50\end{array}$ \\
\hline \multirow[t]{2}{*}{ TLBO } & $\begin{array}{c}\text { Population size : } \\
20\end{array}$ & \multirow[t]{2}{*}{ ITLBO } & $\begin{array}{c}\text { Population size : } \\
20\end{array}$ \\
\hline & $\begin{array}{r}\text { Maximum Number } \\
\text { of Iterations : } 50\end{array}$ & & $\begin{array}{l}\text { Maximum Number } \\
\text { of Iterations : } 50\end{array}$ \\
\hline
\end{tabular}

\subsection{IEEE 118-bus test system}

The proposed method is also applied to IEEE 118-bus system [18]. Congestion is created by outaging the line (85 ) and its effect is given in table 8 . The bids submitted by generators to alter their schedule are given in table 9. As the violation for the line (8-30) is more, the sensitivities are calculated for this line.

The calculated Generator Sensitivity Factors (GSF) and Incremental Generator Sensitivity Factors (IGSF) are presented in table 10. Figure 5, represents the GSF values of various generators in the 118-bus system. It can be seen from table as well as figure that generators numbered 24 and 26 have maximum negative GSF values, and 27 to 36 have maximum positive GSF values. For the economic system operation, it is essential to consider the IGSF values. Finally, the generators numbered 24 and 26 are selected for increasing its real power and the generators numbered 31 , 32,34 and 36 are identified for decreasing its real power for congestion alleviation as these generators having least IGSF values. With this selection, the number of participating generators for the congestion management is only 6 , whereas the total number of generators in the system is 54 .

6.2a Calculation of congestion management cost and rescheduling power: The proposed ITLBO based approach is also implemented to find the congestion cost and active power rescheduling of IEEE 118-bus test 
Table 6. Comparison of results with other algorithms without DSB for IEEE 30-bus test system.

\begin{tabular}{|c|c|c|c|c|c|c|}
\hline Control variables & GA & PSO & $\mathrm{DE}$ & IDE & TLBO & ITLBO \\
\hline$\Delta \mathrm{P}_{\mathrm{G} 1}(\mathrm{MW})$ & -52.64 & -59 & -58 & -54.4 & -56.2 & +20.2 \\
\hline$\Delta \mathrm{P}_{\mathrm{G} 2}(\mathrm{MW})$ & +30.42 & +19.9 & +20.5 & +20.9 & +18.9 & +16.2 \\
\hline$\Delta \mathrm{P}_{\mathrm{G} 5}(\mathrm{MW})$ & +16.66 & +13 & +14.5 & +18.2 & +15.6 & +3.3 \\
\hline$\Delta \mathrm{P}_{\mathrm{G} 8}(\mathrm{MW})$ & +0.65 & +6 & +8 & +7.79 & +6.1 & +8.4 \\
\hline$\Delta \mathrm{P}_{\mathrm{G} 11}(\mathrm{MW})$ & +4.91 & +6.5 & +9.2 & +4.39 & +5.9 & +4.9 \\
\hline$\Delta \mathrm{P}_{\mathrm{G} 13}(\mathrm{MW})$ & +6.2 & +7 & +3.2 & +8.39 & +7.1 & -54 \\
\hline MVA flow on the congested line & 130 & 129 & 130 & 129.89 & 127.91 & 127.66 \\
\hline Congestion Management Cost $(\$ / \mathrm{h})$ & 1561.63 & 1521.80 & 1560 & 1554.05 & 1517.40 & 1486.2 \\
\hline Real power loss (MW) & 15.4 & 15 & 14.4 & 12.6 & 11.8 & 11.512 \\
\hline Execution Time in seconds & 4.2 & 3.4 & 3.7 & 2.8 & 3.2 & 2.6 \\
\hline
\end{tabular}

Table 7. Comparison of results with other algorithms with DSB for IEEE 30-bus test system.

\begin{tabular}{lcccrrr}
\hline Control variables & GA & PSO & DE & IDE & TLBO & ITLBO \\
\hline$\Delta \mathrm{P}_{\mathrm{G} 1}(\mathrm{MW})$ & -4.6 & -3.9 & -4.1 & -3.6 & -2.9 & $+\mathbf{2 5 . 6 1}$ \\
$\Delta \mathrm{P}_{\mathrm{G} 2}(\mathrm{MW})$ & +30.928 & +28.9039 & +28.0863 & +28.0799 & +27.91 & $-\mathbf{2 7 . 6 5}$ \\
$\Delta \mathrm{P}_{\mathrm{G} 5}(\mathrm{MW})$ & -29.8078 & -28.8607 & -27.6032 & -27.5229 & -28.01 & $-\mathbf{1 6 . 6 8}$ \\
$\Delta \mathrm{P}_{\mathrm{G} 8}(\mathrm{MW})$ & -19.5645 & -21.5294 & -19.0257 & -18.60 & -18.00 & $+\mathbf{1 5 . 9 9}$ \\
$\Delta \mathrm{P}_{\mathrm{G} 11}(\mathrm{MW})$ & +18.0108 & +16.9866 & +17.9829 & +17.2431 & +16.10 & $+\mathbf{4 . 9 6}$ \\
$\Delta \mathrm{P}_{\mathrm{G} 13}(\mathrm{MW})$ & +6.0889 & +6.0037 & +7.220 & +4.810 & +5.80 & $-\mathbf{2 . 2 3}$ \\
$\mathrm{MVA}$ flow on the congested line & 130 & 130 & 129.16 & 130 & 129.6 & $\mathbf{1 2 9 . 7 1}$ \\
Congestion Management Cost $(\$ / \mathrm{h})$ & 1380.44 & 1338.02 & 1313.4 & 1266.85 & 1245.95 & $\mathbf{1 1 7 0 . 7 1}$ \\
Real power loss $(\mathrm{MW})$ & 11.34 & 12.00 & 12.05 & 12.65 & 12.00 & $\mathbf{1 1 . 4 5}$ \\
Execution time in seconds & 4.2 & 3.4 & 3.7 & 2.8 & 3.2 \\
\hline
\end{tabular}

Table 8. Line flow on the congested line for IEEE 118-bus test system.

\begin{tabular}{lccc}
\hline $\begin{array}{l}\text { Congested } \\
\text { line }\end{array}$ & $\begin{array}{c}\text { Line flow } \\
\text { (MVA) }\end{array}$ & $\begin{array}{c}\text { Maximum limit } \\
\text { (MVA) }\end{array}$ & $\begin{array}{c}\text { Violation } \\
\text { (MVA) }\end{array}$ \\
\hline $30-17$ & 506.49 & 500 & 6.49 \\
$8-30$ & 419.46 & 175 & 244.46 \\
\hline
\end{tabular}

system. The influence of the ITLBO parameters on the convergence process has been studied and the obtained objective function values with varying TLBO parameters are given in figure 6 . The selected parameters in order to obtain the minimum objective function value are a number of learners: 20 and a maximum number of iterations: 50 .

The results obtained by employing proposed ITLBO for IEEE 118 are listed in table 11. Incorporation of DSB model with the GR for CM reveals a significant reduction in CMC. CMC got reduced to $3344.58 \$ / \mathrm{h}$. The real power change in the slack bus due to the rescheduling process is nil whereas in the previous case, the slack bus needs to be adjusted up to $76 \mathrm{MW}$. This adjustment alone cost about $4560 \$ / \mathrm{h}$ which itself is higher than the cost incurred by the
Table 9. Generator bidding co-efficients for IEEE 118-bus test system.

\begin{tabular}{|c|c|c|c|c|c|}
\hline $\begin{array}{l}\text { 1) } \\
\text { Generator } \\
\text { bus No. }\end{array}$ & $\begin{array}{l}\text { 2) Bids } \\
\text { in } \$ / \\
\text { MWhr }\end{array}$ & $\begin{array}{c}\text { 3) } \\
\text { Generator } \\
\text { bus No }\end{array}$ & $\begin{array}{l}\text { 4) Bids } \\
\text { in } \$ / \\
\text { MWhr }\end{array}$ & $\begin{array}{c}5) \\
\text { Generator } \\
\text { bus No }\end{array}$ & $\begin{array}{l}\text { 6) Bids } \\
\text { in \$/ } \\
\text { MWhr }\end{array}$ \\
\hline 1 & 25 & 42 & 10 & 80 & 18 \\
\hline 4 & 19 & 46 & 20 & 85 & 17 \\
\hline 6 & 16 & 49 & 21 & 87 & 16 \\
\hline 8 & 21 & 54 & 13 & 89 & 15 \\
\hline 10 & 12 & 55 & 18 & 90 & 11 \\
\hline 12 & 13 & 56 & 16 & 91 & 9 \\
\hline 15 & 14 & 59 & 15 & 92 & 10 \\
\hline 18 & 17 & 61 & 17 & 99 & 21 \\
\hline 19 & 19 & 62 & 19 & 100 & 30 \\
\hline 24 & 17 & 65 & 25 & 103 & 15 \\
\hline 25 & 15 & 66 & 27 & 104 & 14 \\
\hline 26 & 17 & 69 & 60 & 105 & 11 \\
\hline 27 & 19 & 70 & 15 & 107 & 20 \\
\hline 31 & 20 & 72 & 14 & 110 & 21 \\
\hline 32 & 15 & 73 & 17 & 111 & 22 \\
\hline 34 & 10 & 74 & 9 & 112 & 23 \\
\hline 36 & 18 & 76 & 6 & 113 & 19 \\
\hline 40 & 14 & 77 & 20 & 116 & 25 \\
\hline
\end{tabular}


Table 10. GSF and IGSF values on the congested line (8-30) in IEEE 118- bus test system.

\begin{tabular}{|c|c|c|c|c|c|}
\hline $\begin{array}{l}\text { Generator } \\
\text { bus No. }\end{array}$ & $G S F$ & $I G S F$ & $\begin{array}{c}\text { Generator } \\
\text { bus No. }\end{array}$ & $G S F$ & $I G S F$ \\
\hline 1 & 0.003 & 83.33 & 65 & -0.009 & -27.77 \\
\hline 4 & -0.004 & -47.50 & 66 & -0.009 & -30.00 \\
\hline 6 & -0.08 & -2.00 & 69 & 0 & 0 \\
\hline 8 & -0.08 & -2.62 & 70 & -0.021 & -7.14 \\
\hline 10 & -0.06 & -2.00 & 72 & -0.042 & -3.33 \\
\hline 12 & -0.06 & -2.16 & 73 & -0.042 & -4.04 \\
\hline 15 & -0.04 & -3.50 & 74 & -0.008 & -11.25 \\
\hline 18 & -0.04 & -4.25 & 76 & -0.007 & -8.57 \\
\hline 19 & -0.04 & -4.75 & 77 & -0.006 & -33.33 \\
\hline 24 & -0.1 & -1.70 & 80 & -0.005 & -36.00 \\
\hline 25 & -0.06 & -2.50 & 85 & -0.004 & -42.50 \\
\hline 26 & -0.33 & -0.51 & 87 & -0.001 & -160.00 \\
\hline 27 & 0.32 & 0.594 & 89 & -0.0004 & -375.00 \\
\hline 31 & 0.51 & 0.392 & 90 & -0.0008 & -137.50 \\
\hline 32 & 0.83 & 0.181 & 91 & -0.0005 & -180.00 \\
\hline 34 & 0.42 & 0.238 & 92 & -0.0006 & -166.66 \\
\hline 36 & 0.52 & 0.346 & 99 & -0.0003 & -700.00 \\
\hline 40 & 0.04 & 3.500 & 100 & 0.003 & 100.00 \\
\hline 42 & 0.06 & 1.667 & 103 & 0.003 & 50.00 \\
\hline 46 & 0.05 & 4.00 & 104 & -0.005 & -28.00 \\
\hline 49 & 0.01 & 21.00 & 105 & -0.005 & -22.00 \\
\hline 54 & 0.006 & 21.66 & 107 & -0.005 & -40.00 \\
\hline 55 & 0.005 & 36.00 & 110 & -0.005 & -42.00 \\
\hline 56 & 0.007 & 22.85 & 111 & -0.005 & -44.00 \\
\hline 59 & -0.002 & -75.00 & 112 & -0.005 & -46.00 \\
\hline 61 & -0.006 & -28.33 & 113 & -0.005 & -38.00 \\
\hline 62 & -0.004 & -47.50 & 116 & -0.005 & -50.00 \\
\hline
\end{tabular}

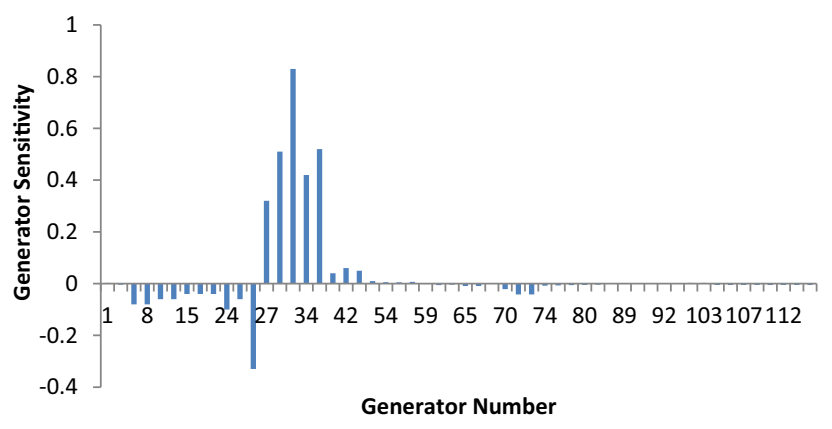

Figure 5. Plot of GSF values verses generator number for IEEE 118-bus test system.

proposed method. Also, it may be noted that with the proposed rescheduling process, the congestion is completely relieved without causing any overload in other lines. The convergence characteristics obtained for the IEEE 118-bus is shown in figure 7. The effect of the proposed DSB model on the real power adjustments at every participating generator is depicted in figure 8 .

6.2b Comparison with other heuristic algorithms: The comparison of the results obtained for IEEE 118-bus by the

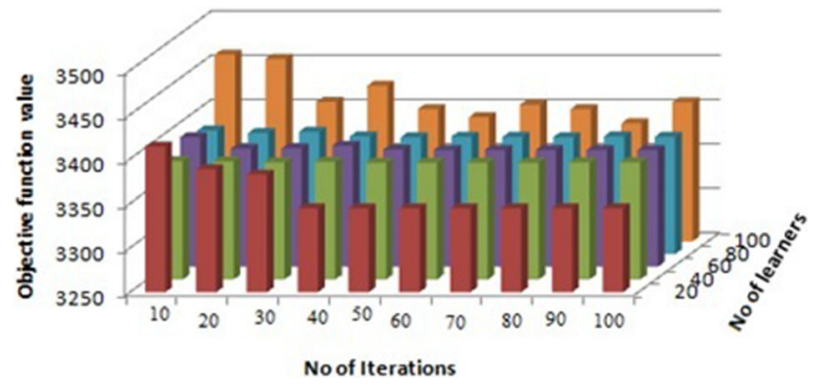

Figure 6. Objective function values for different parameter settings of ITLBO for IEEE 118-bus test system.

Table 11. Optimal settings of decision variables and congestion cost obtained by ITLBO algorithm for IEEE 118- bus test system.

\begin{tabular}{lcc}
\hline & $\begin{array}{c}\text { GR without } \\
\text { distributed slack } \\
\text { bus }\end{array}$ & $\begin{array}{c}\text { GR with } \\
\text { distributed slack } \\
\text { bus }\end{array}$ \\
\hline$\Delta \mathrm{P}_{\mathrm{G} 24}(\mathrm{MW})$ & +64.1 & +24.41 \\
$\Delta \mathrm{P}_{\mathrm{G} 26}(\mathrm{MW})$ & +73.3 & +75.43 \\
$\Delta \mathrm{P}_{\mathrm{G} 31}(\mathrm{MW})$ & -16.4 & -24.12 \\
$\Delta \mathrm{P}_{\mathrm{G} 32}(\mathrm{MW})$ & -16 & -40.14 \\
$\Delta \mathrm{P}_{\mathrm{G} 34}(\mathrm{MW})$ & -13.2 & -10.2 \\
$\Delta \mathrm{P}_{\mathrm{G} 36}$ (MW) & -15.4 & -25.6 \\
$\Delta \mathrm{P}_{\mathrm{G} 69}$ (MW) (Slack & -76 & 0 \\
Bus) & 169.12 & 172.61 \\
$\mathrm{MVA}$ flow on the & $\mathbf{7 8 7 4 . 1 9}$ & $\mathbf{3 3 4 4 . 5 8}$ \\
congested line (8-30) & & \\
Congestion \\
Management Cost \\
$\begin{array}{l}\text { (\$/h) } \\
\text { Real power loss (MW) }\end{array}$
\end{tabular}

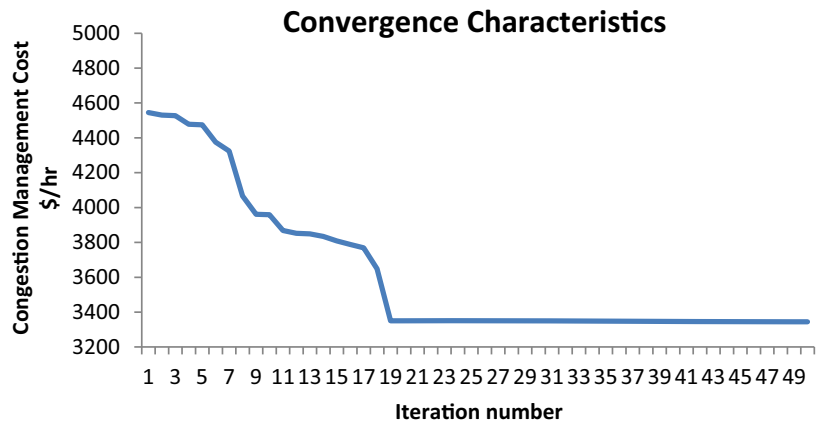

Figure 7. Convergence characteristics of ITLBO with DSB model for IEEE 118-bus test system.

proposed ITLBO algorithm with other heuristics algorithms is shown in table 12. From the table, it may be observed that the CMC is $\mathbf{3 3 4 4 . 5 8} \mathbf{\$} / \mathbf{h}$ by applying the proposed algorithm and it is the lowest one as compared to the other algorithms reported in the same table.

Hence, the proposed algorithm is capable of producing the best result for both the test systems considered. The concept of self-motivated learning introduced in the 
Table 12. Comparison of results with other algorithms with DSB for IEEE 118-bus test system.

\begin{tabular}{|c|c|c|c|c|c|c|}
\hline Control variables & GA & PSO & $\mathrm{DE}$ & IDE & TLBO & ITLBO \\
\hline$\Delta \mathrm{P}_{\mathrm{G} 24}(\mathrm{MW})$ & +64.2 & +81.9 & +44 & +61 & +46.8 & +24.41 \\
\hline$\Delta \mathrm{P}_{\mathrm{G} 26}(\mathrm{MW})$ & +53.2 & +23.49 & +74.7 & +46.2 & +54.9 & +75.43 \\
\hline$\Delta \mathrm{P}_{\mathrm{G} 31}(\mathrm{MW})$ & -26.4 & -21.3 & -28.86 & -34.9 & -26.2 & -24.12 \\
\hline$\Delta \mathrm{P}_{\mathrm{G} 32}(\mathrm{MW})$ & -46 & -55 & -54.3 & -37.8 & -41.2 & -40.14 \\
\hline$\Delta \mathrm{P}_{\mathrm{G} 34}(\mathrm{MW})$ & -13.2 & -9 & -14.42 & -9.6 & -18.3 & -10.2 \\
\hline$\Delta \mathrm{P}_{\mathrm{G} 36}(\mathrm{MW})$ & -25.6 & -16.3 & -15.6 & -23.4 & -18.1 & -25.6 \\
\hline$\Delta \mathrm{P}_{\mathrm{G} 69}(\mathrm{MW})$ (Slack Bus) & -6.2 & -3.79 & -1.52 & -1.5 & -2.1 & 0 \\
\hline MVA flow on the congested line $(8-30)$ & 172.67 & 171.13 & 175 & 170.81 & 170.69 & 169.12 \\
\hline Congestion Management cost $(\$ / \mathrm{h})$ & 4059.8 & 3653.43 & 3925.81 & 3694.6 & 3505.7 & 3344.58 \\
\hline Real power loss (MW) & 5.6 & 4 & 7.1 & 6 & 6.8 & 6.52 \\
\hline Execution time in seconds & 10.3 & 8.7 & 9.2 & 8.2 & 8.00 & 7.6 \\
\hline
\end{tabular}

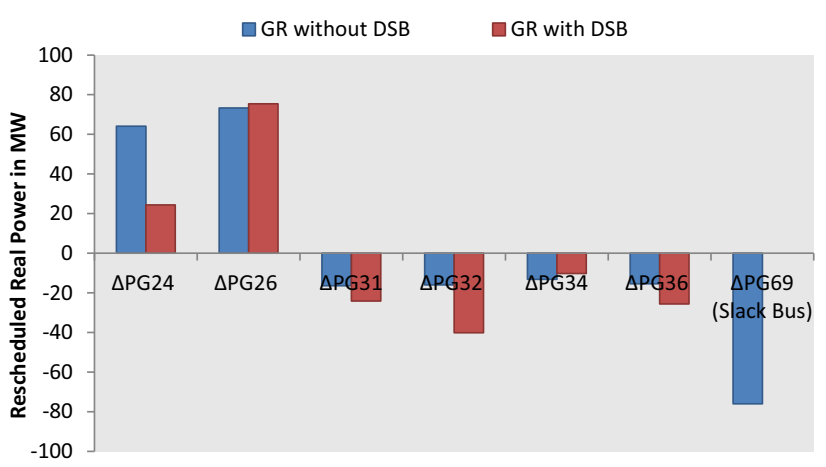

Figure 8. Rescheduled real power with and without DSB for IEEE 118-bus test system.

TLBO algorithm helps in obtaining the better solution as well as faster convergence than the original TLBO algorithm.

\section{Conclusion}

This paper presents a novel method for congestion management through generation rescheduling with distributed slack bus model. Introduction of the power distribution factor in the generator sensitivity helps in distributing the concentrated real power on the slack bus due to the rescheduling process, to all other existing generators proportional to their maximum MW rating. The newly defined incremental generator sensitivity factor is used to identify the most sensitive buses for congestion alleviation. The incremental sensitivity factors ensure the minimum congestion cost by rescheduling the most sensitive buses. The proposed method has been successfully tested on standard IEEE 30-bus system and standard IEEE 118-bus system using ITLBO algorithm. The concept of self-motivated learning introduced in the TLBO algorithm enhances its performance and speed up the convergence process. It has been demonstrated that the combination of ITLBO algorithm and the selection of participating generators for the rescheduling process using the newly defined sensitivity factor yielded better results when compared to other stateof-the-art methods.

\section{Acknowledgements}

This research work was funded by World Bank Group under Robert S McNamara Fellowship Grant.

\section{List of Symbols}

$n \quad$ number of buses in the system

$n_{g} \quad$ number of generator buses in the system

$n_{l} \quad$ number of transmission lines in the system

$P_{i j} \quad$ real power flow through line k connected between buses $\mathrm{i}$ and $\mathrm{j}$

$\Delta \quad$ refers to change in a variable

$R_{g}^{u} \quad$ incremental bidding cost of generator at bus $\mathrm{g}$ in $\$ /$ MW

$R_{g}^{d} \quad$ decremental bidding cost of generator at bus $\mathrm{g}$ in $\$$ / MW

$\Delta P_{G g}^{u} \quad$ incremental real power to be increased on generator at bus $\mathrm{g}$ for congestion alleviation

$\Delta P_{G g}^{d} \quad$ incremental real power to be decreased on generator at bus $\mathrm{g}$ for congestion alleviation

$P_{G g}^{0} \quad$ value of real power generation at generator bus $\mathrm{g}$ resulted in market clearing procedure

$P_{G g}^{\min } \quad$ minimum limit on real power of generator bus $g$

$P_{G g}^{\max } \quad$ maximum limit on real power of generator at bus $\mathrm{g}$

$F_{k} \quad$ apparent power flow in MVA through transmission line $\mathrm{k}$ connected between buses $\mathrm{i}$ and $\mathrm{j}$

$F_{k}^{\max } \quad$ maximum limit of apparent power flow through transmission line $\mathrm{k}$ connected between buses $\mathrm{i}$ and $\mathrm{j}$

$F_{k}^{0} \quad$ apparent power through transmission line $\mathrm{k}$ resulted in market clearing procedure 


\section{References}

[1] Motto A L, 2002 Network-constrained multi period auction for a pool-based electricity market. IEEE Trans. Power. Syst., 17(3): 646-653

[2] Arroyo J M and Conejo A J 2002 Multi period Auction for a pool based Electricity Market. IEEE Trans. Power. Syst., 17(4): 1225-1231

[3] Jiansheng Lei 2001 Congestion management for generator scheduling in a deregulated Chinese power system. In: Proceedings of IEEE PES, Winter Meeting. Columbus, USA

[4] Suganthi S T, Devaraj D and Hosimin Thilagar S 2015 An Improved Differential Evolution algorithm for Congestion Management considering voltage stability. Indian Journal of Science and Technology, 8(24): 0974-6846., September 2015.https://doi.org/10.17485/ijst/2015/v8i24/80243

[5] Talukdar B K, et al 2005 A computationally simple method for cost-efficient generation rescheduling and load shedding for congestion management. Int. J. Elect. Power Energy Syst., 27(5): 379-388

[6] Hazra J, Sinha A K and Phulpin Y 2009 Congestion Management using Generation Rescheduling and/or Load Shedding of Sensitive Bus. In: Proceedings of Third International Conference on Power Systems. Kharagpur, India

[7] Surender Reddy S 2017 Multi-Objective Based Congestion Management Using Generation Rescheduling and Load Shedding. IEEE Transactions on Power Systems, 32(2): 852-863

[8] Leonardo Geo Manescu, et al 2016 Congestion management using dispatch or phase shifting transformers. In: Proceedings of International Conference on Applied and Theoretical Electricity. Craiova, Romania

[9] Singh S N and David A K 2001 Optimal location of FACTS devices for congestion management. Elect.Power.Syst. Res. 58: 71-79

[10] Anwar S. Siddiqu and Tanmoy Deb 2014 Congestion management using FACTS devices. International Journal of System Assurance Engineering and Management, 5(4): 618-627

[11] Tong Shiqiong and Miu K N 2006 Participation factor studies for distributed slack bus models in three phase distribution power flow analysis. In: Transmission and Distribution Conference and Exhibition, IEEE PES. Dallas, TX, USA

[12] Shiqiong Tong and Miu K N 2005 A network-based distributed slack bus model for DGs in unbalanced power flow studies. IEEE Transactions on Power Systems, 20(2): 835-842

[13] Nabavi S M H, Shahram Jadid, Masoum M A S and Kazemi A 2006 Congestion management in nodal pricing with genetic algorithm. In: International Conference on Power Electronics, Drives and Energy Syst. New Delhi, India

[14] Nabavi, S M H, Kazemi, A and Masoum M A S 2011 Social Welfare Improvement by TCSC using Real Code Based Genetic Algorithm in Double-Sided Auction Market. Advances in Electrical and Computer Engineering, 11(2): 99-106

[15] Hazra J and Sinha A K 2008 Congestion management using multi objective particle swarm optimization. In: Proceedings of Power and Energy Society General Meeting - Conversion and Delivery of Electrical Energy in the 21st Century. Pittsburgh, PA, USA

[16] Pandya K S and Joshi S K 2013 Sensitivity and particle swarm optimization based congestion management. Electric Power Components and Systems, 41(4): 465-484

[17] Pal D, Kumar S, Mandal K K and Chakraborty N 2014 Multi-objective congestion management using hybrid differential evolution in a deregulated power system. In: Proceedings of International Conference on Control, Instrumentation, Energy and Communication. Calcutta, India

[18] Preetha Roselyn J, Devaraj D and Subhransu Sekhar Dash 2013 Multi-Objective Differential Evolution for Voltage Security Constrained Optimal Power Flow in Deregulated Power Systems. International Journal of Emerging Electric Power Systems, 14(6): 591-607

[19] Rao R V, Savsani V J and Vakharia D P 2011 Teachinglearning based optimization: a novel method for constrained mechanical design optimization problems. Computer Aided Design, 43(1): 303-315

[20] Rao R V, Savsani V J and Vakharia D P 2012 Teachinglearning based optimization: an optimization method for continuous nonlinear large scale problems. Inf.Sci. 183(1): 1-15 